\title{
VENCEDORAS Y VENCIDAS: LAS EDUCADORAS LEONESAS ANTE LA \\ GUERRA CIVIL ESPAÑOLA
}

Raquel Poy Castro

Universidad de León

rpoyc@unileon.es

Recibido: 03-11-2010

Aceptado: 16-02-2011

\section{Resumen}

A comienzos del siglo $\mathrm{XX}$ un notable número de educadoras españolas tuvieron una importante presencia pública. El artículo examina las tensiones entre el hecho de ser educadora y mujer en la España de 1936-1939 en la ciudad de León. Concluye que este periodo conflictivo impulsó a las educadoras a la acción política apoyando el movimiento de reformas en Educación o, en el lado opuesto, el tradicionalismo conservador. El trabajo relaciona las causas por las que recibieron represión física y política durante y después de la Guerra civil española.

Palabras Clave: Historia de la educación, Guerra Civil Española, España, mujeres, género.

\begin{abstract}
In the early 20th century, large numbers of Spanish women teachers had an important public presence. This article examines the tensions between being a woman and a teacher in the Spain of 1936-1939 in the city of León. It concludes that conflicting times propelled women teachers to political action on key issues such as the support for the reformist movement on education or, in the opposite direction, the conservative traditionalism. The work relates the reasons why they received political and physical repression during and after the Spanish Civil War.
\end{abstract}

Keywords: History of education, Spanish Civil War, Spain, women, gender. 


\section{Introducción}

A comienzos de la Guerra civil, las educadoras que ejercían su labor hasta ese momento empiezan a sufrir la represión impuesta desde ambos bandos del conflicto. De nuevo la espada se volvía más fuerte que la pluma. Por un lado, las facciones sublevadas contra el gobierno de la República cargan inmediatamente contra aquellos que intentaban modernizar la sociedad en la raíz de las ideas, del pensamiento y de las costumbres de una España que había atravesado el siglo XIX entre pronunciamientos y sucesivas crisis económicas, frustrando los intentos de modernización. A estos les culparían de haber generado graves amenazas contra un statu quo que se había mantenido durante décadas en precario equilibrio. Por otro lado, quienes respaldaban la legitimidad republicana cometerían también acciones de represión contra los partidarios de la rebelión. Si en el bando republicano la represión se concentra en los dos primeros años de la contienda, hasta el final del 1937, en el bando franquista se prolongaría durante toda la Dictadura (Casanova, 2007).

Antes de llegar a la fatídica guerra, la década de 1930 presenta para la historia de la educación un escenario privilegiado que permite analizar, comparándola con otros países, la modernización educativa contemporánea española. En los países con regímenes liberales avanzados, la incorporación de las mujeres al movimiento modernizador de la educación se produce desde la década de 1860 hasta finales de 1920. En Estados Unidos la irrupción de las mujeres en los estudios superiores se concentra en las décadas de 1920 y 1930 (Solomon, 1985).

En España, pese al retraso económico propio de los países del sur y este de Europa, las políticas liberales facilitaron una incipiente incorporación femenina a las aulas. Ahora bien, hasta 1910 no se derogan todas las prohibiciones que impedían el acceso de las mujeres a la universidad. Con todo, el papel de las educadoras en el cambio social de España resulta tan importante como innegable desde el siglo XIX, más visible cuando las condiciones del orden republicano les permiten alcanzar notoriedad profesional y política.

La modernización educativa es especialmente notable en la provincia de León por la conjunción de proyectos reformistas que se venían fraguando allí desde las décadas anteriores a la instauración de la República. Se trata esencialmente de una corriente reformista que, retomando antecedentes de la época isabelina, eclosiona en la labor educativa de la Fundación Sierra Pambley, realizada bajo la dirección de la Institución Libre de Enseñanza. Las escuelas de dicha Fundación incorporarían además 
buena parte de los postulados de la nueva pedagogía renovadora que se extendía por Europa en aquellos tiempos (Aguado Cabezas, 2008: 201-234). Lógicamente, la adhesión reformista no sería unánime: otra parte del magisterio provincial, distante de la propuesta anterior, optaría por corrientes conservadoras, católicas e incluso filofascistas, en vísperas de la guerra civil.

En unos y otros grupos el papel de las mujeres será muy notable dentro de la provincia leonesa. A su creciente presencia en las filas del magisterio se une la relevancia profesional, académica y política que algunas de ellas representan en el concierto provincial y estatal, constituyendo una vanguardia intelectual femenina que en gran medida se verá truncada por el episodio bélico de 1936-1939. A ellas va dirigido este artículo.

\section{El contexto de reformas educativas en vísperas de la Guerra Civil}

A comienzos de 1936, la educación y la cultura en España habían alcanzado un notable impulso, fruto de seis décadas de progresivo crecimiento en la actividad y política educativas, que arrancan del periodo de la Restauración (1871-1923). La creación de la Institución Libre de Enseñanza (ILE) en 1876 constituye un importante revulsivo, un punto de partida en dicha evolución. Pero fue necesaria la concurrencia del Estado para que sus propuestas lograran la deseada reforma del panorama educativo español. Esta implicación empezó a dar sus frutos a comienzos del siglo XX, cuando la Administración central se hace cargo del magisterio -hasta entonces competencia de los ayuntamientos, el nivel administrativo más corrupto de la Restauración- y comienza a dignificar la profesión

La creación del Ministerio de Instrucción Pública y Bellas Artes (R. D. 18. 04. 1900) durante la Regencia de María Cristina, del que fue primer titular Antonio García Alix, supone la aparición de una estructura propia y específica en la Administración del Estado, capaz de proponer y ejecutar políticas orientadas al desarrollo educativo. Desde entonces, hasta aproximadamente la década de los años 30, fueron creadas 10.000 escuelas. Además se impulsaron instituciones tan decisivas como la Junta de Ampliación de Estudios (JAE) y se reformaron las Escuelas Normales.

El intervencionismo estatal de esas décadas en materia educativa procede de la colaboración que se estableció entre ministros de la izquierda del partido liberal (Moret, Castrillejo, Romanones) y representantes del institucionismo (Giner de los Ríos y 
Bartolomé Cossío, entre otros). Estos últimos renunciaron parcialmente al principio de una educación totalmente independiente del Estado (dada la debilidad de la sociedad civil española) y orientaron la actuación política del ala izquierda liberal, con la que compartían la idea genérica de modernizar la sociedad española (Moreno Luzón, 2010). Ambas partes coincidían también en que era necesario realizar reformas para evitar la revolución. La educación sería el instrumento de modernización y progreso de España frente a la creciente conflictividad del movimiento obrero que se leía como una amenaza.

Pero sería la proclamación de la Segunda República y la victoria de los partidos progresistas, encabezados por los grupos socialistas y republicanos de izquierda, el hito que daría a la política educativa de comienzos de la década un impulso sin precedentes. El nuevo gobierno republicano abordaba así el reto integral de la instrucción pública en un país con veintitrés millones y medio de habitantes y una tasa de analfabetismo del $32 \%$, así como un millón de niños sin escolarizar. Hasta diciembre de 1931, bajo el mandato del Ministro Marcelino Domingo, de Izquierda Republicana, y con el socialista Rodolfo Llopis al frente de la Dirección General de Primera Enseñanza, se abordaron las primeras medidas urgentes y de mayor espectro reformista.

Estas primeras medidas se centraron en el impulso a la dotación de plantilla y medios para el sistema escolar. Así se daba continuidad también al modelo ideológico regeneracionista, ejemplificado por el lema "escuela y despensa" de Juan Costa, mientras el ideario reformista y pedagógico de la ILE, que analizaba las raíces del atraso socioeconómico español como una consecuencia de la incultura y el atraso rural, alimentaba el discurso de los primeros ministros de Instrucción de la República (Morente, 2001).

El primer gobierno republicano impulsó la mejora salarial de los 36.680 maestros que existían en 1931 y los equiparó a otras categorías de funcionarios. En la práctica ello suponía el ascenso de 14.000 maestros, dando prioridad a los que padecían una situación más precaria (Pérez Galán, 1977). El Decreto de 24 de junio creaba de una sola vez 7.000 plazas de nuevos maestros y maestras para las escuelas nacionales. Hasta el final de la Segunda República, se había abierto un promedio de 3.200 plazas anuales de maestros, frente a las 962 anuales de la década de 1920 (De Puelles, 2008).

En segundo lugar, se impulsó la creación de centros. Al proclamarse la República existían 35.716 escuelas en España, apenas 11.000 más que veinticinco años antes, con un promedio de 500 centros nuevos al año, de modo que el gobierno republicano se marcó como objetivo la creación de 27.000 nuevas; consiguió crear 
7.000 sólo en el primer año (Navarro García, 2002), pero este ritmo no se mantuvo constante en todo el periodo republicano.

Paralelamente existía la preocupación prioritaria de elevar la cualificación del profesorado. El Ministerio de Instrucción Pública señalaba que en 1926 existían 10.501 maestros ejerciendo la enseñanza privada, incluyendo educadores religiosos y seglares, de los cuales el 57,9 carecía de titulación que los habilitase para la docencia. El objetivo central era acabar tan lamentable situación (Llopis, 1933).

Otras disposiciones de los primeros meses de gobierno republicano servirían para reforzar la capacidad ejecutiva del nuevo sistema: se abordaba una reforma de las Escuelas Normales donde se formaba a los maestros y maestras, y se creaba el Patronato de Misiones Pedagógicas bajo la presidencia de Manuel Bartolomé Cossío, sucesor de Francisco Giner de los Ríos y líder de los institucionistas. Como es bien conocido, las Misiones Pedagógicas llevarían la creación de bibliotecas escolares a todo el territorio, organizando una campaña de expediciones educativas a zonas rurales y de difícil acceso, bajo el propósito de la alfabetización cultural de una amplia capa de población desprovista de atención educativa o cultural. El peso de tan ambicioso proyecto recaía en los maestros y maestras, así como en el cuerpo de Inspección, que ahora adquiría un nuevo carácter.

A comienzos de la Guerra ya se habían superado los 43.000 maestros, junto a 2.051 profesores de instituto, 1.357 profesores de escuelas especiales y 1.233 profesores universitarios. Aunque la reforma continuó avanzando hasta julio de 1936, el gobierno conservador (1933 y 1935) ralentizó el ritmo de creación de centros y sobre todo detuvo la implantación de la coeducación.

En León, la acción decidida de la Inspección educativa se unió al esfuerzo de la red institucionista representada por la Fundación Sierra Pambley. Ello multiplicó los recursos y facilitó el alcance de las acciones del Patronato, como las inversiones en infraestructura, que permitieron disponer de una red bibliotecaria escolar y rural, o la organización exitosa de diversas Misiones pedagógicas en los pueblos de la provincia.

\section{Del moderantismo a la reacción frente a las reformas: conservadurismo y tradicionalismo educativos}

Las manifestaciones intelectuales que componen el tradicionalismo constituyen una expresión amplia de la ideología que defiende la conservación de las costumbres, 
destacadamente católicas y populares, como instrumento de recuperación del orgullo patriótico, especialmente impulsado durante la década primorriverista, a imitación de la ola nacionalista europea y francesa en particular. Pero también, para los Kraussoinstitucionistas era básica la nacionalización del país a través de la enseñanza de la Historia y el Patrimonio artístico español. En amplias esferas de las clases medias, entre las que se venían reclutando los docentes desde mediados del siglo XIX, había calado una honda simpatía por estos asuntos, a veces leídos en clave conservadora y otras en clave reformista. En León se puede observar el desarrollo de ambas corrientes.

La profesora Vega Rodríguez (2006) ha estudiado lo que denomina la "hora regionalista" leonesa de la década de 1920, a través de la prensa y la actividad local. En su opinión entronca con una moda populista que reivindica el cancionero popular, como lo haría la Generación poética del 27, y que también se inspiraría en la labor etnológica de Ramón Menéndez Pidal. Entre los intelectuales locales más significativos, se incluiría el leonés Publio Suárez que en un artículo de 1924 se lamentaba de la pérdida de identidad, amenazada por la industrialización, el olvido de la cultura tradicional y el costumbrismo como seña de identidad. Otros autores leoneses, como Jacinto Rojo, secundarían este movimiento local, si bien los mayores exponentes del mismo serían los catedráticos y sucesivos directores del Instituto de León, Mariano Domínguez Berrueta y, con antelación a éste, Juan Eloy Díaz-Jiménez, gran recuperador del patrimonio arqueológico y eclesiástico leonés, y padre de una de las protagonistas de nuestro artículo.

Si el tradicionalismo había sido el caldo de cultivo de ideas conservadoras y nacionalistas durante las décadas anteriores, a ello se unió la radicalización de las posturas reaccionarias a las reformas republicanas, particularmente en torno al conflicto con la Iglesia católica, agudizado por la persecución al clero, el incendio de conventos, y las medidas laicistas que pretendían romper con el secular monopolio eclesiástico de la educación. En vísperas de 1936, el problema religioso serviría de estímulo en la polarización de las filas conservadoras. Y es que, frente a los graves problemas y las prioridades de la reforma educativa de los gobiernos republicanos, una de las medidas más polémicas en la sociedad española fue la que emanó del decreto de 1931 que suprimía la obligatoriedad de la instrucción religiosa en las escuelas. Las reacciones del clero y de importantes colectivos sociales fueron virulentas y se llegó a convertir en una cuestión de Estado, al menos hasta el surgimiento de la Revolución de Asturias de 1934. Además, el segundo ministro de educación republicano, Fernando de los Ríos -sobrino de Francisco Giner de los Ríos - adoptó como primera actuación la expulsión de la Orden de los Jesuitas en enero de 1932, lo que ahondaría el problema relativo a la 
“cuestión religiosa", y todo ello pese a que bajo su mandato continuó el impulso a la financiación de nuevas escuelas y se abordaron profundas reformas legislativas de la estructura educativa.

En torno a estos grupos que rechazan parte de las medidas del nuevo gobierno republicano, se agruparán una buena parte de aquellos intelectuales y docentes que han compartido en las últimas décadas el ideario tradicionalista, para lo cual se produjo un movimiento de reacción en buena parte de la intelectualidad y la prensa conservadoras, y fue impulsado por los gobiernos del bienio derechista.

Las vencedoras autoridades franquistas les reclutarían masivamente para la dirección de las instituciones educativas, a fin de ejecutar la nueva política de represión y contrarreforma educativa, pues constituyeron la alternativa lógica a los desaparecidos pedagogos progresistas. No en vano, la ideología configurada por el franquismo haría del tradicionalismo católico una de sus señas de identidad más importantes.

\section{Del moderantismo a la reacción frente a las reformas: conservadurismo y tradicionalismo educativos}

\subsection{Inspección de Primera Enseñanza: la élite educativa en la década de 1930}

La Inspección de Primera Enseñanza es una figura de carácter esencial para comprender la reforma de la estructura educativa en España. Su condición de instrumento de ejecución de una política educativa centralizadora -por cuanto su misión esencial es la aplicación de las leyes de educación y preservar la homogeneidad de la acción educativa conforme a las directrices del Estado- será recurrente bajo todos los gobiernos centralistas desde el siglo XVIII.

El papel de la inspección en la Segunda República adquirió cotas sin precedentes en cuanto a importancia ejecutiva. El institucionismo concibió la Inspección como una especie de Escuela Normal a domicilio o de profesorado ambulante, idea que plasmó F. Giner de los Ríos desde 1900 a través del Boletín de la Institución Libre de Enseñanza (BILE, 1933: 237-295). Decía Giner que el maestro urbano o rural necesitaba del Inspector como "alguien que vea de cerca su obra, pueda compararla con la de los otros, le advierta y aconseje, no como «jefe», sino como un amigo, como un compañero más experimentado".

Tres competencias centrales, que les fueron asignadas a los Inspectores por el Decreto de 1932, tendrían un alcance sin precedentes. En primer lugar la edición de un 
Boletín de Educación, que tendría una función ilustrativa integral para el docente, abarcando diversos ámbitos pedagógicos, organizativos y técnicos. El segundo instrumento fue la elaboración de documentación reguladora, como los cuestionarios a utilizar en las Escuelas y los Reglamentos de las instituciones escolares de Primera Enseñanza. La tercera, la misión dinamizadora del colectivo docente, tendría un alcance especial en lo que suponía el proyecto de responsabilidad que debían imprimir los inspectores en la labor educativa. Para ello se preveía la constitución de Juntas locales de inspectores que debían organizar cursillos de información pedagógica, viajes de estudio, conferencias, etc., con la finalidad de "interesar y orientar al Magisterio en los problemas de la educación". También preveía un conjunto de iniciativas dirigidas al fomento de "la creación de Centros de colaboración pedagógica, agrupando en ellos a maestros de pueblos próximos que se reúnan periódicamente para estudiar aspectos concretos de la vida escolar; hacer lecciones modelo seguidas de crítica; adquirir mancomunadamente el material; promover actos públicos en favor de los intereses de la Escuela, etc.". Así mismo contemplaba la contribución de la Inspección a la organización de Museos pedagógicos y Bibliotecas circulantes en su demarcación.

La Inspección de Enseñanza era también un instrumento institucionista por la influencia que ejercían los docentes de la Escuela Normal central de Madrid o Escuela Superior de Formación del Profesorado, donde se formaba a esta élite educadora. Como señalaba Juan Comas Camps - coautor del Manual del Inspector de Primera Enseñanza con Rafael Álvarez y Juvenal de Vega, quien había sido alumno entre 1918 y 1921 de la Escuela de Estudios Superiores del Magisterio, y había vivido en la Residencia de Estudiantes- a los inspectores les inculcaban, más que una serie de competencias, el objetivo de:

“[...] suprimir la idea de que un inspector es un señor que está sentado en una esquina vigilando que el maestro entre a las ocho en punto y que salga a las doce en punto, y que haga cosas mecánicas; y que, al contrario, fuera un señor que se pusiera en plan de maestro, de enseñar con el maestro, de encontrar dificultades, porque ninguno de nosotros era ningún sabio, ni ningún hombre perfecto. Habíamos leído un poco más, habíamos visto más cosas que el maestro rural, pero la idea era, y lo conseguimos en mi generación, que el inspector fuera un compañero más que ayudara a los pobres maestros rurales1" (Gómez y Romero, 2006: 11).

Por la Inspección de León pasarían algunos de los más insignes y destacados inspectores. A la cabeza de todos ellos Rafael Álvarez García, uno de los coautores del Manual referido, natural de Villablino, e Inspector Jefe provincial, con su secretario Manuel F. Fierro, junto a 18 inspectores y funcionarios docentes. Interesa aquí destacar 
a las mujeres inspectoras: Matilde Trebol, Francisca Bohigas, Purificación Merino, Estefanía González, Julia Morros, Francisca Vicente, María Santos y Concepción Ares.

El destino de este cuerpo de inspectores fue variado. Tras la Guerra, tres serían fusilados (Rafael Álvarez, José Ruiz y Luis Vega), una moriría de las secuelas (Francisca Vicente), diez serían destituidos de la docencia y una trasladada (Francisca Bohigas).

\section{Vencedoras y vencidas: las educadoras en el León de 1936}

El papel de las educadoras en la década de 1930 destaca por una intensidad sin precedentes en los diferentes planos de la vida política, económica y social. En el primer cuarto del siglo XX las educadoras adquieren su máximo nivel de conciencia científica y transformadora de la sociedad a través de la profesión. Es frecuente encontrarlas compatibilizando su acción pedagógica con la política y social: militan en partidos políticos, sindicatos, asociaciones y todo tipo de movimientos sociales; esto incluye la participación en sociedades y grupos de carácter masónico, católico o tradicionalista, tanto en ideologías progresistas como conservadoras o de orientación extremista. No obstante la presencia de mujeres en las élites profesionales e intelectuales es aún muy minoritaria, aunque en el ámbito educativo como en el conjunto de profesiones de carácter "asistencial" se localiza la mayor presencia femenina desde el siglo XIX.

En el marco leonés, podemos agrupar a las educadoras en alguno o varios de los grupos o modelos pedagógicos que conviven en la década de 1930, y que hacen de esta provincia un auténtico laboratorio social, reflejo de la ebullición sociopolítica nacional. Un primer modelo es el "institucionista", desarrollado por la ILE y por todas las instituciones que creó. En León esta corriente está representada por la Fundación Sierra Pambley. Un segundo grupo aparece alineado con posiciones de la izquierda republicana y con la escuela anarquista. El tercer modelo, que denominaríamos conservador tradicionalista, encarna ocasionalmente el reaccionarismo: Defienden lo que consideraban derechos educativos de la iglesia, contra unas políticas educativas que consideraban conducían a la revolución.

Finalmente, un cuarto grupo podría resumir una corriente conservadora moderada, que defiende postulados en el marco del cristianismo social, en su día alineado con una parte del maurismo, y, en León, con el datismo (partidarios de Eduardo Dato), que había sido partícipe de algunas de las reformas de las dos décadas anteriores. En León corresponde a este grupo la discreta iniciativa educativa que en la 
Restauración impulsaron miembros de la familia Álvarez Carballo, después Fundación del mismo nombre.

\subsection{Educadoras y vencidas}

Un movimiento pedagógico, social e institucional, más o menos organizado, reúne a educadores y educadoras partidarios del reformismo institucionista y los elementos republicanos, liberales o progresistas, partidarios de la Escuela Nueva. Sus simpatizantes y partidarios se suman indistintamente o a la vez a partidos políticos, asociaciones y organismos que promueven las reformas educativas, con mayor o menor empeño.

En los años previos a la Guerra Civil, este grupo leonés de reformistas y progresistas, se aglutinan en particular en la Fundación Sierra Pambley, la mayoría de ellos discípulos de Bartolomé Cossío, Giner de los Ríos y del leonés Gumersindo de Azcárate, fundadores de la ILE. Entre todos ellos destacan por su ingente labor organizativa e intelectual algunos nombres clave: Rafael Álvarez García, Nicostrato Vela, Luis Vega, Pío Alvarez, Herminio Almendros y Rafael Santamaría, y tres mujeres, las inspectoras María Cuyás y Francisca Vicente, junto con la profesora María Pedrosa.

Este grupo de educadores, no sólo inspectores o profesores de las Escuelas Normales, también manifestaron una adhesión mayoritaria al programa reformistas de la República -la mayoría serían depurados a partir de 1936-, bien por convicción de sus ideas o por simple obediencia hacia el amplio despliegue de tales actividades. La acción política de este grupo de profesores y profesoras fue muy intensa en esos años.

\subsubsection{Julia Morros}

Julia Morros Sardá refleja las especiales dificultades que padecieron en particular las mujeres dentro del movimiento pedagógico de la época. Se trata de una representante esencial en el conjunto de educadoras que optaron por la Inspección, y que junto a sus compañeros formados en la Escuela Superior del Magisterio, recibieron sin duda el estímulo de las enseñanzas paidológicas y se interesaron por la nueva psicología, aún cuando después derivaran hacia otros estudios o especialización (Carpintero y Herrero, 2007).

Nuestra protagonista tenía 34 años cuando estalla la Guerra Civil, y destaca por ser una leonesa pionera en el ámbito de la pedagogía y la higiene escolar (Moreno Martínez, 2007). Licenciada en Ciencias Naturales y diplomada como Maestra Nacional, fue número uno de la $14^{\mathrm{a}}$ promoción de la Escuela de Estudios Superiores del 
Magisterio (1922-1926), donde había iniciado una prometedora carrera docente como profesora auxiliar de clases prácticas de Fisiología, Higiene Escolar y Antropometría Pedagógica. Más tarde sería Catedrática de la Escuela Normal de Madrid y profesora en el Instituto Escuela Especial de Psicología Experimental e Higiene.

Fue una de las 26 personas pensionadas por la JAE para realizar estudios relacionados con la Higiene escolar, de las cuales 17 eran pedagogos. Durante diez meses de estancia en Francia y Bélgica estudió antropometría, que le serviría para adquirir una adecuada cualificación investigadora. En Francia estuvo en el Instituto de Psicología y Pedagogía de la Sorbona, organizado en 1920 para la especialización en materias de psicología, ${ }^{1}$ (Carpintero y Herrero, 2007: 210). También estudió en el Institut de Psychologie (París), bajo la dirección de M. Pieron y M. Meyerson, así como en L'École de Anthropologie, En Ginebra pasó por el Instituto J. J. Rousseau para estudiar con E. Claparède y P. Bovet.

Es autora del estudio «Valores y relaciones cefálicas de las niñas madrileñas» (JAE, microficha, M-124), trabajo previo a su Tesis doctoral, sobre "El crecimiento en la edad escolar. Estudio antropométrico comparativo de niños leoneses", una investigación que sería publicada por la Sociedad Española de Antropología, Etnografía y Prehistoria de Madrid en 1935.

Todo lo anterior convirtió a Julia Morros en la sucesora natural del prestigioso profesor Luis Hoyos (también pensionado de la JAE y catedrático de Fisiología e

\footnotetext{
${ }^{1}$ Por Real Decreto del 11 de enero de 1907, y bajo el ministerio liberal de Amalio Gimeno, se crea la Junta para Ampliación de Estudios e Investigaciones Científicas (JAE), que tendrá un efecto revolucionario en la formación del personal docente y científico español, muy especialmente a través de su política de becas (1907-1936). Su primer Presidente fue Santiago Ramón y Cajal (1852-1934), galardonado el año anterior con el premio Nobel, y ejerció dicho papel junto a José Castillejo, su Secretario durante tres décadas. Algunos de sus primeros consejeros fueron Gumersindo de Azcárate, Leonardo Torres Quevedo, José Echegaray, Marcelino Menéndez y Pelayo o Ramón Menéndez Pidal. Sus fines comprenden los siguientes: «a) El Servicio de ampliación de estudios dentro y fuera de España. b) Las delegaciones en congresos científicos. c) El servicio de información extranjera y relaciones internacionales en materia de enseñanza. d) El fomento de trabajos de investigación científica. e) La protección de las instituciones educativas en la enseñanza secundaria y superior». Entre sus creaciones destacan centros de investigación como el Centro de Estudios Históricos y el Instituto Nacional de Ciencias Físico-Naturales con todos sus laboratorios, y centros educativos como la Residencia de Estudiantes. Pese a sus relaciones difíciles tanto con los gobiernos de Primo Rivera como republicanos, perdurará hasta 1936. En 1938 el Ministerio de Educación Nacional disuelve la JAE y crea en su lugar el Consejo Superior de Investigaciones Científicas. entre 1907 y 1934 la Junta concedió 1594 becas de estancia en el extranjero, individuales y en grupo, de las que más del 50\% correspondieron a maestros y profesores para estudios pedagógicos, y otro 38\% a médicos. En el caso de los pensionados en Pedagogía, un total de 280 recibieron becas para realizar estudios individuales o en grupo, con una ratio de 97 mujeres (un 34,63\% del total) frente a 183 varones (Moreno Martínez, 2007: 174).
} 
Higiene de la Escuela de Estudios Superiores del Magisterio desde 1909), de quien había sido alumna muy aventajada. Al parecer, renuncia por motivos económicos a la carrera docente, atraída por la retribución y estabilidad profesional que promete el ejercicio como Inspectora de Primera Enseñanza. Cuando estalla la guerra se encuentra ejerciendo como tal en León, lo que resultará determinante en la depuración posterior (Carpintero y Herrero, 2007).

Carpintero y Herrero la ubican en el exilio en Argentina tras la Guerra, pero fuentes de la Consejería de Educación de Castilla-La Mancha señalan que posteriormente le permitieron reincorporarse a la docencia y ejerció durante varios años en Guadalajara (1957-1964) como profesora de Ciencias de la Escuela Normal de Magisterio, donde llegaría a desempeñar el cargo de Directora de esta misma Escuela, y desde donde aún escribiría libros escolares de enseñanza de las Ciencias² .

Julia Morros es el ejemplo de una mujer docente e investigadora, víctima de la depuración, que vio truncada su carrera científica y docente universitaria en Madrid. El exilio primero, y la resignación a una oscura carrera docente en provincias, a su regreso, supusieron una de las trayectorias representativas de quienes no resultaron vencedoras en el conflicto.

\subsubsection{Nieves González Barrio}

El caso de esta segunda docente e investigadora, también resulta ilustrativo de las dificultades con las que se enfrentaron los intelectuales españoles en el ámbito educativo e investigador para conseguir formación continua y especializada. Leonesa de clase media, realizó estudios universitarios y fue una de las dos primeras mujeres en graduarse como médicas. Nieves había nacido en 1884 en una familia de maestra y comerciante de Vegacervera, y defendió brillantemente su tesis doctoral en 1915. Posee un impresionante currículo, que incluye algunos libros publicados antes de la Guerra Civil, e incluso al término de la misma publica su libro 'Guerra a los microbios'

Agudizado por el hecho de adelantarse en varios años al periodo aperturista republicano, Nieves González insiste en ejercer la medicina dentro de un sistema diseñado para hombres de modo exclusivo, el cual la castiga asignándole un destino profesional terrible en la ciudad africana de Tetuán, bajo protectorado español, y al servicio del harén de mujeres del Jalifa, monarca del lugar. Sin embargo no renuncia a

\footnotetext{
${ }^{2}$ Obras de Julia Morros sobre pedagogía son las siguientes: Problemas de Física y Química. Ed. Juvenal. Madrid, 1954.; Iniciación a la Química. Ed. Juvenal. Madrid, 1954; El Museo de Ciencias Naturales en la Escuela Primaria. Madrid.
} 
su vocación, y consigue una beca de la Junta de Ampliación de Estudios para estudiar en París. A partir de entonces su carrera científica será imparable, al acceder a prestigiosos laboratorios de ciudades como Chicago o Nueva York en los que estuvo trabajando (Fernández León 2008).

El suyo sería un exilio forzado por la necesidad de encontrar un ámbito en el que poder desarrollar su talento.

\subsubsection{María Cuyás Pons}

Inspectora y esposa de Herminio Almendros, María Cuyás obtiene un puesto docente en la Fundación Sierra Pambley con su marido y ambos se desplazan a Villablino. Junto a Herminio contribuye esencialmente a la introducción de los nuevos métodos pedagógicos; ella es la traductora de Robert Dottrens (1934).

Introductores de Freinet en España y Latinoamérica, su labor teórica tiene una especial significación, pese a que la estancia de ambos en León, como profesores de la Fundación Sierra Pambley, no fue demasiado larga. Pero la relevancia pedagógica de ambos en España, justifica su inclusión en este grupo de renovadores de la pedagogía en el León Republicano.

Tras su labor docente en las Escuelas leonesas de Sierra Pambley, se convierten en inspectores de primera enseñanza en las provincias de Lérida (1928), Huesca (1931), donde junto a militantes libertarios como Ramón Acín y Félix Carrasquer, organizarían ese mismo año el I Congreso Nacional de Maestros, y posteriormente se trasladarían a Barcelona (1933). En 1936 se exilian en Cuba, desde donde continuarán con su labor científica y pedagógica (Hernández Díaz, 2001). Particularmente a Herminio, su ideario institucionista y su defensa de la escuela popular le procuran una notable fama como escritor de literatura infantil en su época. Constituye un referente esencial de la pedagogía defensora de la escuela rural renovada y activa, para afianzar una cultura inserta en las necesidades reales de sus habitantes. María y Herminio comparten su labor intelectual y participan activamente en las Misiones Pedagógicas, como las del Valle de Arán, donde mantienen una intensa relación con otros activos colaboradores de las mismas, como Alejandro Casona, con quien comparten sus inquietudes por los ambientes culturales y sociales desfavorecidos.

Durante su largo exilio en Cuba, el matrimonio tendrá ocasión de ejercer el magisterio y mantener su labor científica en las siguientes décadas. 


\subsection{Educadoras y vencedoras}

Están alineadas en la órbita conservadora y tradicionalista que reúne inicialmente a las partidarias del proyecto político conservador encarnado en los años 20 por la Unión Patriótica de Primo de Rivera. Un proyecto nacionalista que ensalza el patriotismo militarista y que concibió algunas medidas de modernización, pero hostil al proyecto de abierto cambio que promoverían los gobiernos de izquierdas de la Segunda República. Las figuras esenciales de ese grupo en León son la diputada Francisca Bohigas, Mariano Domínguez Berrueta, y algunos miembros menos notables como José María Vicente y Mercedes Monrroy, directores respectivamente de las Escuelas Normales de Maestros y Maestras. Aunque en 1936 será sometido al proceso general de depuración, pues había realizado numerosas adhesiones al régimen republicano, Domínguez Berrueta demostraría su fidelidad al nuevo régimen, colaborando como inquisidor oficial del colectivo docente. Se le otorgó el papel de responsable de la depuración de la propia Fundación Sierra Pambley. Posteriormente, el régimen le concedió numerosos reconocimientos y se convirtió en el cronista oficial de León.

Pero el caso más sobresaliente de este grupo conservador es el alcanzado por María Díaz-Jiménez, profesora de la Escuela Normal y militante del movimiento educativo y católico teresiano. Discípula aventajada de su fundador, en 1939 recibirá el alto cometido de dirigir la Escuela Normal central en Madrid, a la que llegará a dar nombre hasta la fecha actual.

\subsubsection{Mercedes Monrroy}

Hasta la unión en virtud de la aplicación del principio de coeducación según el decreto del 29 de septiembre de 1931, la Escuela Normal disponía de dos directores: José María Vicente para la masculina, y Mercedes Monrroy para la de maestras (Gaitero, 2006).

Durante el siglo XIX, sostenidas en precario por las Diputaciones Provinciales, las Escuelas Normales habían languidecido, y sólo en la década de 1920 recuperaban el aliento con la incorporación a los Presupuestos del Estado. Estas reformas rescataron a la Escuela Normal de León de la dependencia económica de la Diputación provincial, lo que supuso una mayor dependencia de Madrid, pero menor de la presión local. Un claustro reducido de diez profesores permitió sostener su actividad formativa y la reforma de 1932 proporcionó una inyección de recursos y nuevas competencias como centro de formación continua del profesorado. 
La Segunda República les confiere el mandato de actuar como centro dinamizador de la red de escuelas rurales y, con el Decreto de 1931, las Escuelas Normales de hombres y de mujeres en cada ciudad se refundieron en una sola, adquiriendo un papel central en la formación y apoyo a la labor del Magisterio nacional. En 1933, la Orden de 17 de abril, disponía la organización de cursos de perfeccionamiento en las Escuelas Normales, destinados al apoyo al enseñante, especialmente el de las zonas rurales, "protegiéndole y estimulando eficazmente su renovación espiritual", con una duración de dos semanas cada cuatrimestre, y donde debía colaborar la Inspección provincia. No eran sólo cursos profesionales, también incluían temas científicos, literarios, culturales y especialmente relativos al mundo rural español. Además, la Escuela Normal de León desarrollaba en ese momento una notable actividad pública, con cursos destinados a la formación de docentes y actuando también como foco cultural mediante la impartición de cursos de extensión dirigidos a la divulgación y formación popular.

Mercedes Monrroy era la directora de la Escuela Normal de Maestras, miembro activo de la Unión Patriótica desde su formación, y con presencia en las iniciativas asociadas a la misma, como la Sociedad de Estudios Leoneses impulsada por significados miembros de dicho partido (Vega Rodríguez, 2006).

Pero, de modo similar a los educadores alineados con los partidos más progresistas, aquellos que asumieron posiciones más moderadas no se libraron de ser sometidos a los procesos de depuración y, en muchos casos, sufrir represalias.. Mercedes sería depurada tras el conflicto bélico y apartada de la dirección de la Escuela.

\subsubsection{Francisca Bohigas Gavilanes}

León tuvo una destacada presencia de políticos e intelectuales alineados con el conservadurismo social. El agrario fue el de mayor alcance político, pues consiguió importantes resultados electorales en las convocatorias del periodo republicano. También el líder de la CEDA (Confederación Española de Derechas Autónomas), José María Gil Robles - reivindicando su origen leonés-, encabezaría la candidatura por la provincia al Congreso y, junto a él, una de las escasas candidatas que accedieron al Congreso: la Inspectora de Primera Enseñanza Francisca Bohigas. Frente a las ocho candidatas de las izquierdas que obtuvieron acta de diputadas durante el periodo republicano, fue la única elegida por los partidos conservadores (Álvarez, 2005). Formaba parte de un partido político integrado exclusivamente por mujeres, la Acción Femenina Leonesa, fundado en 1931 con ideario católico, tradicionalista y agrario, y 
que se había coaligado con la CEDA. Procedente de un destino en Lérida, en 1928 se integró en la Unión Patriótica impulsada por Primo de Rivera.

Francisca Bohigas jugó un destacado papel durante la República como una de las máximas representantes del proyecto tradicionalista educativo en España, pues su posición como diputada nacional desde 1933 confirió una amplia repercusión a su labor, ejemplificada en sus conferencias y mítines en diversos puntos urbanos y rurales, y su presencia constante a través de la prensa. Estuvo enfrentada con Rafael Álvarez y el grupo de colegas reformistas se mantuvo a lo largo de todo el periodo republicano.

En un primer momento, Francisca se alineó claramente con los vencedores. Al estallar la rebelión armada, Bohigas no dudó en saludarlo como una buena noticia para la educación. En agosto de 1936 manifestaba lo siguiente: «mientras el Ejército y demás instituciones armadas junto con las milicias derrama su sangre joven en el frente de batalla y ofrendan sus vidas llenas de promesas y esperanzas para salvar el presente de España y la civilización cristiano occidental, los Maestros, con la colaboración de las autoridades lucharemos aquí contra la invasión ideológica extranjera y atea, y ofreceremos a España, perfectamente sana, la generación del provenir» (Serrano Fernández y Álvarez Oblanca, 1987).

Sin embargo, su incesante labor y preocupación por restaurar la normalidad educativa, como sucedió al reclamar el abandono de los militares de las aulas ocupadas en el complejo escolar Ponce de León, le granjean el ostracismo. Fue trasladada a Sevilla por diferencias personales con el jefe de la Comandancia de la Guardia Civil.

La represión de Bohigas es quizás la más paradójica, y la que representa con mayor dureza la crueldad de un nuevo régimen en el que las libertades y derechos se suprimieron sin consideración para las mujeres, independientemente de los méritos de los que se hubieran hecho acreedoras.

\subsection{3. $M^{\mathrm{a}}$ Rosario Díaz Jiménez}

Si existe una auténtica vencedora entre las educadoras más prestigiosas del León de 1936, esa es María Díaz Jiménez. Hija de Juan-Eloy Diaz-Jimenez y Villamor, catedrático de Psicología, Lógica y Ética en el Real Instituto de León, carlista y estudioso de la historia local, que había desarrollado su autor de numerosas obras sobre arqueología y patrimonio religioso de la ciudad. Renunció a ser preceptor de Alfonso XIII por razón de la militancia carlista de su familia, adscrita al tradicionalismo católico y seguidora del pensador Balmes. 
María Díaz es una activa colaboradora del movimiento educativo católico representado por los internados Teresianos, que se relacionan con la Junta de damas católicas y promueven la catequesis a través de un amplio movimiento propagandista ${ }^{3}$. La Institución Teresiana abrió academias por todo el país, bajo el liderazgo del sacerdote Pedro Poveda, con quien María mantuvo una estrecha relación, y que fue canonizado en 2003 junto a Escrivá de Balaguer. El movimiento "Acción católica de la mujer en España" fue el eje asociativo que en paralelo a la acción educativa promovió este movimiento.

María formó parte del entorno de la Academia teresiana de León, que se había fundado en 1917 para atraer a las alumnas que cursaban estudios en la Normal, en la de Comercio o que preparaban el ingreso en Bachillerato; es decir, un grupo elitista destinado a encabezar las posiciones educativas en la ciudad, particularmente a través de la Residencia de normalistas, donde el Obispo y diferentes sacerdotes que colaboraban con el centro ejercían una gran influencia.

El 18 de diciembre de 1920, María Díaz participa en Madrid en la primera Asamblea del Profesorado de Normales, en la que se registraron 19 profesoras cooperadoras de la Institución Teresiana entre los 40 asistentes en total, y que formaban parte de la Asociación Misionera de la Institución Teresiana (Paz Velázquez, 2002: 138). Este movimiento se centró particularmente en la promoción de la catequesis femenina.

En junio de 1939 se incorpora como profesora de la Escuela Normal de Madrid y en breve el nuevo régimen la nombró Directora de la Escuela Normal núm. 2 (Escuela Femenina) de Madrid, encabezando la Comisión depuradora de los alumnos que solicitaban continuar estudios en el nuevo curso 1939-1940. Además, se incorpora en la cúspide de la estructura organizativa del aparato depurador ejercido a través de la Comisión de Cultura y Enseñanza, que inicialmente presidió José María Pemán,. María Díaz sería la única mujer del primer grupo de asesores de una Comisión Central formada íntegramente por hombres (Reyes y De Paz, 2009: 345). La Comisión coordinaba la red de Comisiones de Depuración provinciales.

En los listados enviados por María Díaz a la Dirección General de Enseñanza, sus calificaciones resultantes de la depuración de alumnos eran significativas. Los alumnos se agrupaban en varias categorías: "completamente afectos al Movimiento", donde podía aparecer algún alumno destacado por su actuación durante la República;

\footnotetext{
${ }^{3}$ Correspondencia entre el Padre Poveda y María Díaz Jiménez, Jaén, 5 de agosto de 1919 AHIT. Citado en Paz Velázquez (2002).
} 
"totalmente desafectos" en los que se destacaban a los "peligrosos"; los "conversos", es decir, "alumnos que durante el Movimiento rectificaron su mala conducta anterior .poniéndose decididamente al lado de la España de Franco"; de "tendencia izquierdista pasiva” y, por último, los “dudosos” (Morata Sebastián, 2006).

\section{La dramática represión de 1936}

La depuración de los docentes comenzó el 18 de julio, bajo la tutela de los militares, los gobernadores civiles y los rectores simpatizantes de la rebelión, para pasar a ser sistematizada por las Órdenes del Gobierno de Franco de 10 de noviembre de 1936 y 17 de febrero de 1937 (Negrín, 2007).

El proceso, se extendió hasta la década de 1960, y fueron expulsados de la enseñanza 6.000 docentes. La purga actuó sobre la totalidad del cuerpo profesional, aplicando el procedimiento habitual del régimen: todos debían demostrar su 'inocencia', dentro de un explícito proceso de depuración. Cuando el 20 de noviembre de 1975 murió Franco, todavía un conjunto de maestros republicanos fueron rehabilitados en su puesto en la enseñanza.

José María Hernández Díaz (2001) estima que el alcance de la depuración (asesinados, encarcelados, retirados provisional o definitivamente del servicio, exiliados) fue aproximadamente de un $20 \%$ del total del censo de funcionarios del Ministerio de Instrucción Pública, aunque en algunas provincias la tasa se aproxima al $30 \%$, como sucede en Barcelona, con especial énfasis en los profesores de las Escuelas Normales por la alta influencia entre los docentes.

El profesor Morente Valero estima por su parte en un promedio del $26 \%$ los maestros sancionados del conjunto en 17 provincias analizadas con unos 6.000 docentes apartados definitivamente de la enseñanza y unos quince mil depurados de algún modo.

Presidiendo este panorama sancionador, los miembros de la Comisión C se encontraban satisfechos y orgullosos:

\footnotetext{
"Hemos estimado el alto honor que se nos ha dispensado al ser designados para esta empresa patriótica, de depuración moral, y pedimos a Dios que hayamos acertado, y que nuestros desvelos, nuestras pequeñas contrariedades, puedan servir algún día de acicate para más grandes empresas; y que la enseñanza, patrimonio espiritual de todas las generaciones, comience a dar los frutos abundantes de nuestro siglo de oro y etapas de gloria, que siempre fueron envidiadas por las demás naciones" (Morente Valero, 2001: 200).
} 
La Comisión, poniendo a Dios por testigo, dejaba destituida hasta la disolución a toda la plantilla de inspectores, y sancionaba en mayor o menor medida al $80 \%$ de los profesionales de los centros de su competencia (un 10\%, apartados definitivamente de la enseñanza). Por lo que respecta a la Comisión D, instruyó más de 900 expedientes entre 1937 y 1943, y sólo 189 fueron habilitados para la enseñanza o repuestos en sus cargos. Otro capítulo donde la represión ideológica revistió caracteres obsesivos fue el tratamiento dado a las bibliotecas (Aracil y Segura, 2006). Además de la incautación de las bibliotecas escolares que había enviado el Patronato de Misiones Pedagógicas, el resto de las bibliotecas fue convenientemente expurgado, llegándose a instruir a los alcaldes para su destrucción sistemática (Lafoz, 2007).

En términos absolutos, 483 de los 1.405 profesores de Instituto de segunda enseñanza españoles (un 38\%) recibieron algún tipo de sanción entre febrero de 1937 , fecha en la que aparecen las primeras resoluciones sancionadoras, y octubre de 1943, fecha en la que aparecen las últimas sanciones:

Gráfico 1. Sanciones a profesorado de Segunda Enseñanza por el régimen Nacional (1937-1943)

\begin{tabular}{|l|r|r|}
\hline \multicolumn{1}{|c|}{ Resultado de la depuración } & España & \multicolumn{1}{|c|}{ León } \\
\hline Separación del servicio & 205 & 6 \\
\hline Suspensión por un año o más & 31 & 1 \\
\hline Suspensión por menos de un año & 13 & 1 \\
\hline Traslado & 72 & 1 \\
\hline Inhabilitación & 162 & 16 \\
\hline Confirmación en el cargo & 922 & 1 \\
\hline TOTAL & 1.405 & 1 \\
\hline
\end{tabular}

Fuente: Elaboración propia en base a Aracil y Segura (2006: 92)

En los primeros meses tras la rebelión militar, más de 40 docentes fueron fusilados en León, y un primer grupo de 102 maestros fueron destituidos en 48 horas por el Gobernador civil Ignacio Estévez Estévez. En octubre era el Rector de Valladolid, Isidoro de la Villa Sanza, quien cesó a otros 298 maestros, incluyendo 87 de los afectados por la anterior destitución. En el momento de la apertura de curso, eran 500 las vacantes en las plazas de maestros de León.

En cuanto a la actuación de la Comisión Depuradora D para el magisterio e instrucción primaria, establecida el 8 de noviembre de 1936, resolvió 250 expedientes 
en 1937, 58 en 1938 y más de 500 maestros sólo en 1939, afectados por más de 100 modalidades distintas de sanción. En 1943, terminadas las inspecciones, de más de 900 expedientados sólo 189 fueron habilitados para la enseñanza o repuestos en sus cargos (Álvarez Oblanca y Serrano Fernández, 1987).

Los inspectores de enseñanza, posiblemente porque habían recibido la encomienda de conducir y vigilar el proceso reformista republicano, fueron los que recibieron un trato más encarnizado, siendo todos depurados, incluida Bohigas. Todos los directores de centros fueron cesados, con excepción del de la Escuela de Comercio, Arturo García Hidalgo. Asimismo fueron incluidos en la depuración los nueve centros privados de enseñanza de la provincia.

\subsection{Las educadoras a partir de la Guerra civil}

El papel al que se relega la función docente femenina, una vez suprimida la Inspección, se concentra en la labor docente en los centros bajo los nuevos principios y restricciones pedagógicas, que primaban la autoridad en el aula y la recuperación de los tradicionales métodos del manual y la mnemotecnia. En el caso leonés un ejemplo del nuevo modelo pedagógico lo representan las Escuelas agrupadas en el Colegio Ponce de León, fundadas en febrero de 1935 en las antiguas dependencias de la Escuela Normal de Maestros de la C/ Serranos, reuniendo diversos grados escolares femeninos que hasta entonces estaban distribuidos por diversos locales de la ciudad, pero que no inician su actividad hasta septiembre de 1936, ya bajo la autoridad del gobierno sublevado. En total son siete las profesoras y una directora para atender a 504 niñas matriculadas. En sus actas de 1936 se recoge fielmente la nueva situación que afrontan las docentes leonesas en la enseñanza primaria:

“- El primer acto académico consiste en que el párroco de Santa Marina entroniza el crucifijo en las distintas aulas, figura que había desaparecido durante la etapa republicana, lo que obligó a la Escuela a realizar la adquisición de crucifijos al efecto.

- La labor educativa se complementa con tareas de corte y confección, entre las niñas mayores, que les permite elaborar como aguinaldo para el ejército franquista siete pares de calcetines, 16 pasamontañas de lana y 12 pares de calzoncillos confeccionados por las propias niñas. También se aportan 52 pesetas que han logrado recaudar a través de una colecta.

- Al comienzo de cada jornada escolar, se realiza el canto del himno nacional así como el desfile ante la bandera, y las principales fiestas escolares celebrarán el día de la Raza (12 de octubre) la Fiesta del Pilar, los aniversarios de la muerte del general Mola y del político Calvo Sotelo.

- La programación escolar se complementa con la realización de ejercicios espirituales en la iglesia de Santa Marina, impartidos por el padre Hidalgo. 
- Algunas maestras del Colegio serán asimismo represaliadas. La maestra $\mathrm{D}^{\mathrm{a}}$. Isabel Álvarez Álvarez será sancionada por la Comisión de Magisterio con el traslado a otra escuela, por izquierdista, antirreligiosa, desafecta al régimen actual, simpatizante y propagandista de Izquierda Republicana y por haber llegado a León de forma irregular". El Sr. Inspector de Enseñanza informaba al Ayuntamiento que $D^{a}$. Isabel Álvarez llegó a León con plaza en Sierra Pambley por influencia del Sr. Inspector D. Rafael Álvarez (que fue fusilado) y por ser esposa de D. Federico Toral, oficial de Correos, que también había sido suspendido de empleo y sueldo, aunque posteriormente se le repuso en su cargo.

- La Profesora Julia Zapatero Blanco también fue suspendida de empleo y sueldo durante un año, y en febrero de 1940, la Comisión de Magisterio de la provincia de Burgos, pide informes de ella. El 15 de febrero de 1940, el Guarda Municipal, D. Cayo Pérez, dará buenos informes sobre ella" (Actas del Colegio Público Ponde de León, 1936).

El desolador panorama se completa, como señala Pilar Ballarín (2001), con un atraso educativo general para el colectivo de mujeres que se plasmará en el conjunto del franquismo, comenzando por la abolición de la educación mixta de modo radical (Ballarín 1993).

\section{Conclusiones: ¿hubo alguna educadora vencedora?}

El balance de las vencidas por la Guerra es abrumador en el caso de las mujeres, con excepción de María Díaz Jiménez, que durante cuatro décadas ha dado nombre a la Escuela de Magisterio de la Universidad Complutense de Madrid, y aún hoy recibe su nombre el edificio central de la Facultad de Educación.

El caso de Francisca Bohigas, vencedora en los primeros momentos de la sublevación, fue probablemente uno de los más representativos de que los vencedores no toleraban una actitud insumisa en ningún caso. Su enfrentamiento con el oficial al mando de la Guardia Civil en León le causó el traslado forzoso a Sevilla. Sus desvelos iniciales por recuperar la normalidad de los centros escolares, como en el caso de la Escuela Ponce de León, ocupada inicialmente por tropas franquistas, le granjearon la represalia más contundente de los vencedores.

En general, las educadoras más representativas del panorama leonés, de uno y otro signo ideológico, recibieron una dura represión, y durante décadas no se reunió una pléyade de mujeres que brillaran con tanto esplendor como en la denominada Edad de Plata de la educación española, truncada en 1936. 
Además de esta selección de destacadas educadoras que coinciden en vísperas de la Guerra en León, otras muchas sufrirán el silencio de la historia en sus represaliadas carreras profesionales. Así, en noviembre de 1936, entre los maestros de la institucionista Fundación Sierra Pambley se incluyen las depuraciones de la prestigiosa educadora y directora de la Fundación Sierra Pambley María Pedrosa junto a su compañero Bautista R. Calleja, y se fusila a Nicostrato Vela y Pío Álvarez, respectivamente Secretario y Bibliotecario de la institución (Gaitero, 2006). Además, la Fundación vio confiscados sus bienes durante las siguientes cuatro décadas.

Entre las inspectoras que estuvieron destacadas en León en los años previos a la Guerra, cabe mencionar a María de los Dolores Ballesteros Usano, que estuvo becada por la Junta de Ampliación de Estudios para visitar en Francia, Bélgica y Suiza las organizaciones de párvulos y maternales. Hermana de Antonio Ballesteros Usano, gran reformista de la educación infantil e Inspector general durante la Segunda República (Martin Eced, 1991: 55-56).

El resultado principal del nuevo régimen político franquista es la reclusión y confinamiento de las mujeres al espacio doméstico y las profesiones asistenciales (educación, sanidad) para las cuales se supone una predisposición innata y conveniente a los intereses de la sociedad antropocéntrica tradicionalista. Se produce lo que Claudia Goldin (1994: 17) denomina exclusión profesional de la mujer tras la excepción de un periodo bélico, y que se materializa en la limitación de su acceso a la formación, y en consecuencia a la posibilidad de alcanzar retribuciones salariales como los varones, negándosele cualquier expectativa de movilidad social. Otras medidas de reducción de derechos sociales-durante las siguientes cuatro décadas necesitarán permiso de sus esposos para poder disponer de cuentas bancarias - completarán un panorama sombrío para la mujer hasta la llegada de la democracia a finales de la década de 1970. 


\section{BIBLIOGRAFÍA}

- Actas del Colegio Público Ponce de León (1936) [en línea] Disponible en: http://ceipponcedeleon.centros.educa.jcyl.es/sitio/upload/hISTORIA_DEL_CEIP_ PONCE_DE_LEON.pdf [01/11/2011].

- Aguado Cabezas, E. (2008): "Segundo Sierra Pambley y Fernando de Castro. Dos liberales leoneses en la era isabelina”. En F. Carantoña Álvarez y E. Aguado Cabezas (eds.): Ideas reformistas y reformadores en la España del siglo XIX. Madrid: Editorial Biblioteca Nueva, pp 201-234.

- Álvarez Domínguez, J. M. (2005): "Mujeres en política durante la II República. Acción Femenina leonesa a través de la prensa (1931-1936)". En Revista Tierras de León. $\mathrm{n}^{\mathrm{o}}$. 120 y 121, enero-diciembre, pp. 256-285.

- Álvarez Oblanca, W. y Serrano Fernández, S. (1987): “La represión ideológica: las depuraciones”. En Revista Tierras de León. №. 67, Junio, pp. 87-96.

- Aracil Martí, R. y Segura, A. (2006): Educació, municipis i República. Edicions Universitat Barcelona y Diputació de Barcelona. Xarxa de Municipis.

- Ballarín Domingo, P. (2001): La educación de las mujeres en la España contemporánea (siglos XIX y XX). Madrid: Síntesis.

- Ballarin, P. (1993): “El mito de la coeducación”. En J. Ramos Garcia (comp.): El camino hacia una escuela coeducativa. Cuadernos de cooperación educativa, 3. Publicaciones M.C.E.P. Sevilla, pp. 119-128.

- BILE - Boletín de la Institución Libre de Enseñanza (1933). Dirigido por Ricardo Rubio.

- Carpintero, H. y Herrero, F. (2007): "La Junta para Ampliación de Estudios y el desarrollo de la psicología española. Asclepio". En Revista de Historia de la Medicina y de la Ciencia, vol. LIX, nº. 2, julio-diciembre, pp. 181-212.

- Casanova, J. (2007), República y guerra civil, V. III (Historia de España dirigida por J. Fontana y R. Villares). Barcelona: Crítica.

- De Puelles Benítez, M. (2008): Modernidad, republicanismo y democracia: una historia de le educación en España (1898-2008). Valencia: Editorial Tirant lo Blanch. 
- Dottrens, R. (1934): La enseñanza de la escritura: nuevos métodos. Madrid: EspasaCalpe.

- Fernández León, F. (2008): “Modélicas 'bichos raros"”. En La Crónica de León, 19 de noviembre [en línea] Disponible en http://www.la-cronica.net/2008/11/19/ vivir/modelicas-bichos-raros-17563.htm [02-12-2011].

- Gaitero, A. (2006): “Maestras y políticas, costureras y peinadoras". Diario de León, 09 de diciembre [en línea] Disponible en http://www.diariodeleon.es/noticias/ noticia.asp?pkid=295461 [22/01/2011].

- Giner de los Ríos, F. (1933): "El problema de la educación nacional y las clases «productoras»”. En F. Giner de los Ríos: Obras completas. Madrid: Espasa-Calpe, pp. 237-295.

- Goldin, C. (1994): "Understanding the Gender Gap: Ann Economic History of American Women". En P. Burstein (ed.): Equal employment opportunity: labor market discrimination and public policy. New York: Aldine de Gruyter, pp. 17-26.

- Gómez, A. L. y Romero, J. (2006): “Las Comisiones de Instrucción Primaria, el Cuerpo de Inspectores y la difusión de innovaciones educativas en la formación práctico-teórica del profesorado. Avances en supervisión educativa". En Revista de la Asociación de Inspectores de Educación de España, nº. 3, maio, pp. 1-14.

- Hernández Díaz, J. Ma. (2001): "Maestros, inspectores y pedagogos en el exilio español de 1939”. En J. M. Balcells y J. A. Pérez Bowie (eds.). En El exilio cultural de la Guerra Civil, 1936-1939. Salamanca: Eds. Universidad de Salamanca, pp. 95-110.

- Lafoz Rabaza, H. (2007): Aniquilar la semilla de Caín. La represión del magisterio republicano. Zaragoza: Universidad de Zaragoza.

- Llopis, R. (1933): La revolución en la escuela. Madrid: Aguilar.

- Martín Eced, T. (1991): Innovadores de la educación en España: becarios de la junta para ampliación de Estudios.Toledo: Universidad de Castilla La Mancha.

- Morata Sebastián, R. (2006): "Las depuraciones políticas en la escuela normal no 2 de Madrid desde el inicio de la guerra civil hasta los albores del régimen franquista". En Revista Complutense de Educación, vol. 17, nº 1, pp. 153-168. 
- Moreno Luzón, J. (2010): “De cómo fue posible la Residencia”. En Boletín de la Institución Libre de Enseñanza (BILE), (II época), diciembre, pp. 107-118.

- Moreno Martínez, P. L. (2007): “Los pensionados de la Junta para Ampliación de Estudios e Investigaciones Científicas (JAE) y la Higiene Escolar”. En Revista de Educación, número extraordinario, pp. 167-190.

- Morente Valero, F. (2001): "La muerte de una ilusión: el Magisterio español en la Guerra Civil y el primer franquismo". En Historia y Comunicación Social, nº 6, pp. 187-201.

- Navarro García, C. (2002): "La figura del maestro en la Escuela de la República". En Revista Interuniversitaria de Formación del Profesorado, nº. 43, abril, pp. 21-37.

- Negrín Fajardo, O. (2007): “Los expedientes de depuración de los profesores de Instituto de Segunda Enseñanza resueltos por el Ministerio de Educación Nacional (1937-1943)". En Hispania Nova: Revista de Historia Contemporánea, nº. 7, pp. 441457 [en línea] Disponible en http://hispanianova.rediris.es [02/11/2011].

- Paz Velázquez, F. (2002): Sal de tu tierra. Cuadernos biográficos Pedro Poveda, núm. 7. Madrid: Nárcea.

- Pérez Galán, M. (1977): La enseñanza en la Segunda República española. Madrid: Cuadernos para el diálogo.

- Reyes Santana, M. y De Paz Sánchez, J. J. (2009): La represión del Magisterio republicano en la provincia de Huelva. Huelva: Diputación de Huelva.

- Serrano Fernández, S. y Alvarez Oblanca, W. (1987): "El Frente Popular y las elecciones de 1936". En Revista "Tierras de León”, no. 67, junio, pp. 35-45.

- Solomon, B. M. (1985): In the Company of Educated Women: A History of Women and Higher Education in America. New Haven (CT): Yale University Press.

- Vega Rodríguez, P. (2006): “Artículos de etnografía y costumbres en la prensa leonesa: canciones y cantares (1922-1928)". En Culturas Populares. Revista Electrónica, $\mathrm{n}^{\circ} .2$, mayo-agosto, pp. 1-11. 